\title{
Microcontroller Based Heart Beat Monitoring and Alerting System
}

\author{
Mayank Kothari \\ Assistant ProfessorNMIMS, MPSTMEShirpur, India
}

\begin{abstract}
This paper describes the development of a heart beat monitor system based on a microcontroller. The paper explains how a single-chip microcontroller can be usedto analyze heart beat rate in real-time. In addition, it allows doctors to get the heart beat and location of the patient by GSM every twenty four hours. It can also be used to control patients or athletic person over a long period. The system readsstores and analyses the heart beat repetitively in real-time. The hardware and software design are oriented towards a single-chip microcontroller-based system, hence minimizing the size.
\end{abstract}

Keywords: Microsystems, microcontroller, real-time, heart rate monitoring, GSM, GPS

\section{Introduction}

Heart rate is the number of heartbeats per unit of time, typically expressed as beats per minute. Heart rate can vary as the body's need to absorb oxygen and excrete carbon dioxide changes during exercise or sleep.

The measurement of heart rate is used by medical professionals to assist in the diagnosis and tracking of medical conditions. It is also used by individuals, such as athletes, who are interested in monitoring their heart rate to acquire maximum efficiency. Changes in lifestyle

and unhealthy eating habits have resulted in a dramatic increase in incidents of heart and vascular diseases. Furthermore, heart problems are being increasingly diagnosed on younger patients. Worldwide, Coronary heart disease is now the leading cause of death. Thus, any improvements in the diagnosis and treatment tools are welcomed by the medical community.

In a clinical environment, heart rate is measured under controlled conditions like blood measurement, heart beat measurement, and Electrocardiogram (ECG). However, there is a great need that patients are able to measure the heart rate in the home environment as well. The heart rate rises gradually during exercises and returns slowly to the rest value after exercise. The rate at which the pulse returns to normal is an indication of the fitness of the person

This paper presents the design and implementation of a compact and low cost microcontroller-based portable system used for monitoring of heart beat on real time and alerting about patient to a care person in real time.

\subsection{Hardware}

\section{System Specification}

Microcontroller PIC16F877A

$>$ Regulated Power Supply of 5 Volt

$>$ Crystal Oscillator of $8 \mathrm{MHz}$

$>$ Heart Beat Sensor (sunrom-11570)

$>$ GSM Modem(SIM300)

$>$ GPS Receiver(Progin MR-87)

2.2 Software

GPS enabled mobile

$>$ mikroC_8_2_0_0

$>$ PPPv3

$>$ Google Earth

\section{System Block diagram}

The hardware design is based on an embedded system implementation using the PIC16F877A microcontroller from microchip [10].It is 40 bit controller. 


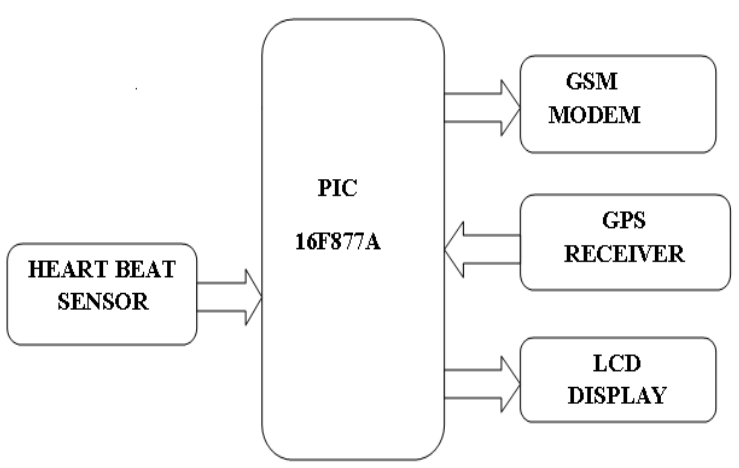

Fig-1

4.1 Proposed Heart beat measuring technique

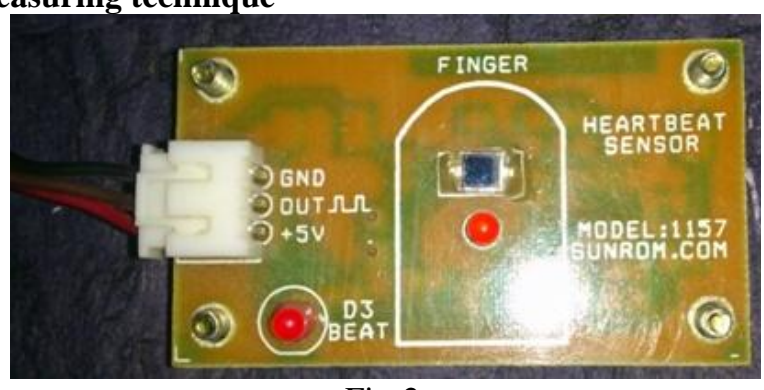

Fig-2

This paper demonstrates a technique to measure the heart rate by sensing the change in blood flow in a finger a while the heart is pumping the blood. This change is measured with the help of heart beat sensor. This sensor consists of light detector and 660nm super red led. Concept behind this $660 \mathrm{~nm}$ led is that the average wavelength of cell tissue in the human body ranged between 600 nanometers and $720 \mathrm{~nm}$; 660 is the mid-point. So in essence, the reason a $660 \mathrm{~nm}$ works better than any other single frequency is because it is closer to the resonant frequency of cell tissue. The other reason is that $660 \mathrm{~nm}$ absorbs better in hemoglobin.

\subsection{Method used to implement}

This system consist of Microcontroller (PIC16F877A), heart beat sensor, GSM modem, GPS receiver. For measuring Heart beat, input is taken from figure of human. Heart beat sensor will generate digital pulse corresponding to each beat. This pulse is counted by interfacing heart beat sensor to microcontroller to pin no. 15(T1CKL) and programming the microcontroller in counter mode. After counting of pulse for one minute, value of heart beat will be displayed on LCD and if value is beyond the normal range then location of patient will be message to care person using GSM. This message contains location in format of latitude and longitude. By inserting this co-ordinate in GPS navigator then you will get exact location of patient.

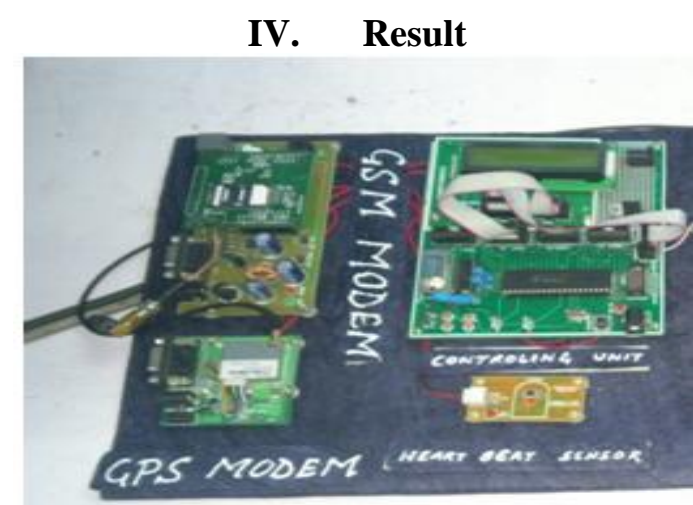

Fig-3 


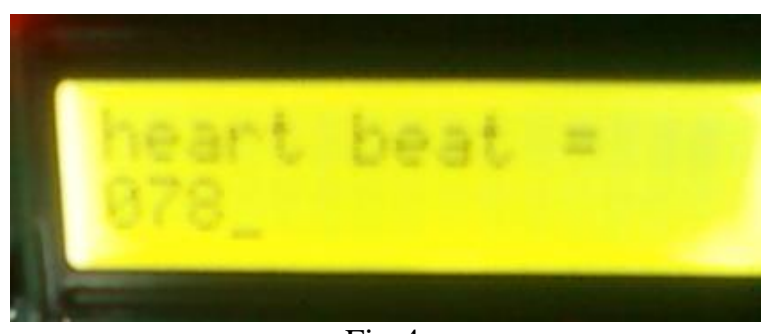

Fig-4

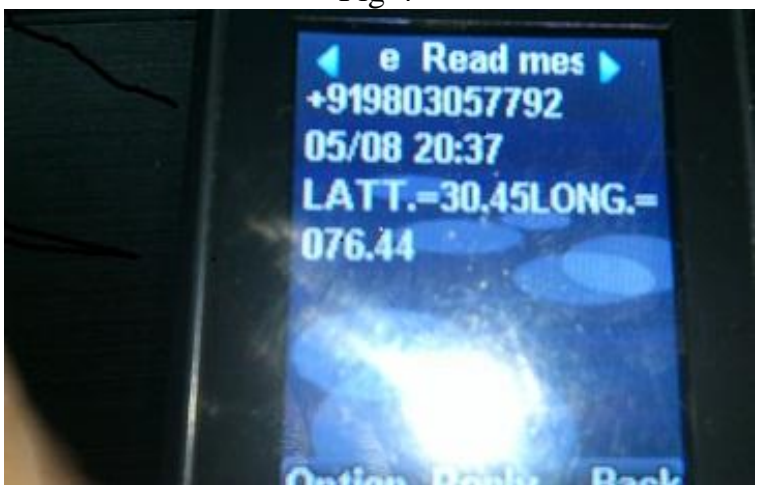

Fig-5

\section{Conclusion}

We have developed the system in such a way that it can be implemented for monitoring heart beat in real time. This system is helpful for Monitoring of Heart beat for athletes and person who are doing exerciseregularly.This system can be used in home, or during travelling, or in hospitals also. For testing this system we have measures heart rate and pressure of different person. By keeping value outside normal range, I checked it will message to care person that message contains latitude and longitude. By inserting these latitude and longitude in Google Earth we got name of location of patient.

\section{References}

[1] Adams, T. D., Yanowitz, F. G., Fisher, A. G., Ridges, J. D., Lovell, K. and Pryor, T. A., 1981, $\square$ Noninvasive Evaluation of Exercise Training in College-Age Men $\square$ Circulation 64: 958-965.

[2] Blair, S.N., Goodyear, N. N., Gibbons, L. W. and Cooper, K. H., 1984, $\square$ Physical Fitness and Incidence of Hypertension in Healthy Normotensive Men and Women $\square$ JAMA 252:

[3] Daniel HE ,(DEC2005) $\square$ Sim 300 AT command set user manual $\square$, Simcom ltd.

[4] Dogan Ibrahim, KadriBuruncuk, $\square$ heart rate measurement from the finger using a low cost microcontroller $\square$, Near EastUniversity, Faculty Of Engineering, TRNC

[5] Jean-Marie Zogg( march 2002),GPS basis, u-bloxZuercherstrasse.

[6] M.J. Burke, M.V. Whelan, $\square$ The Accuracy and Reliability of commercial heart ratemonitor $\square$ Vol. 21, No. 1, March 1987, pp. 29-32.

[7] MichaeleJ.Pont (2002) Embedded C, Pearson education ltd.

[8] Mohamed Fezari, MounirBousbia-Salah,and MouldiBedda, $\square$ Microcontroller Based Heart Rate Monitor $\square$, The International Arab Journal of Information Technology, Vol. 5, No. 4, October 2008.

[9] www.google.com/GoogleEarth/download

[10] www.progin.com/product/mr-87/product Specification

[11] www.sunrom.com/sensor/heart beat sensor/datasheet 